\title{
Bone-Derived Extracellular Vesicles: Novel Players of Interorgan Crosstalk
}

\author{
Yi Li ${ }^{\dagger}$, Pengbin Yin ${ }^{\dagger}$, Zhongkui Guo, Houchen Lv, Yuan Deng, Ming Chen, Ya Gu, \\ Peifu Tang* and Licheng Zhang* \\ Department of Orthopedics, General Hospital of Chinese PLA, Beijing, China
}

\section{OPEN ACCESS}

Edited by:

Andrea Del Fattore,

Bambino Gesù Children Hospital (IRCCS), Italy

Reviewed by:

Michela Rossi,

Bambino Gesù Children Hospital (IRCCS), Italy

David M. Findlay,

University of Adelaide, Australia

*Correspondence:

Peifu Tang

pftang301@163.com

Licheng Zhang

zhanglcheng218@126.com

tThese authors have contributed equally to this work

Specialty section:

This article was submitted to Bone Research

a section of the journal

Frontiers in Endocrinology

Received: 26 July 2019 Accepted: 20 November 2019 Published: 10 December 2019

Citation:

$L i Y$, Yin P, Guo Z, Lv H, Deng Y, Chen M, Gu Y, Tang P and Zhang $L$ (2019) Bone-Derived Extracellular Vesicles: Novel Players of Interorgan Crosstalk. Front. Endocrinol. 10:846 doi: 10.3389/fendo.2019.00846
An increasing number of studies have shown that bone plays an active role in regulating glucose metabolism, affects renal, and cardiovascular diseases and even influences the development of offspring. These novel findings have indicated that bone plays a much more important role in the human body than only providing physical support. However, further investigations of the mechanisms underlying the effects of bone are needed. Recently, extracellular vesicles (EVs) have received increased attention because they can transfer functional proteins, mRNAs, and miRNAs between cells/organs. After reviewing the existing evidence, we hypothesized that bone may be involved in interorgan communication via EVs. Further research exploring bone-derived EVs may facilitate the understanding of bone as a multifunctional organ.

Keywords: extracellular vesicles, interorgan crosstalk, bone factors, osteocalcin, FGF23 = fibroblast growth factor 23, exosomes

\section{INTRODUCTION}

As research has progressed, the functions of various organs have been updated. The common function of bone is traditionally believed to be that of an effector. Bone is usually a target regulated by other organs for physiological function, and to an extent, bone acts as a recipient during physiological communication. However, recent studies have proven that bone not only acts as an effector but also regulates other organs by secreting biological molecules. For example, fibroblast growth factor 23 (FGF23) secreted from osteoblasts and osteocytes can regulate phosphate and mineral metabolism $(1,2)$. Bone-derived osteocalcin $(\mathrm{OCN})$ and lipocalin-2 (LCN2) regulate glucose metabolism and improve insulin resistance. In spite of its metabolic functions, OCN could cross the blood-brain barrier and promote learning and memory (3-5). Receptor activator of NF- $\mathrm{BB}$ ligand (RANKL) and its receptor Receptor activator of NF-kB (RANK) are essential regulators of bone remodeling. Studies have shown that RANKL and RANK are also expressed in the central nervous system and have unexpected functions in controlling inflammation in ischemic brains $(6,7)$. These results strongly suggest that bone has more functions that were ignored by previous research. Bone may regulate the function of other organs under physiological conditions. However, thus far, this novel function of bone has not been fully clarified, as studies generally focus on specific proteins. Few new factors were found to further reveal the characteristic features of bone. The mechanisms underlying these regulatory functions remain a mystery.

Recent studies have found that various organ systems can exchange information between cells through extracellular vesicles (EVs), which is a new mechanism of communication between cells and organs. Studies have found that EVs from different sources are widely involved in endocrine, tumor microenvironment, and nervous system regulation (8-10). These findings indicate that EVs could provide a means for remote tissue communication. Although few studies have found that bone-derived EVs can participate in interorgan communication, the role of EVs involved in 
intercellular communication during bone metabolism has been widely discovered. EVs have been identified as having important functions in regulating the communication between osteoblasts and osteoclasts. The most important discovery is that osteoclastderived exosomal miR-214-3p could be transferred to osteoblasts to inhibit osteoblast activity $(11,12)$. Studies have shown that osteoblast-derived EVs could also contain mRNAs that contribute to the RANKL pro-osteoclastic effects (13). Since bone-related EVs have been found to be important bioactive components involved in bone regulation, are bone-related EVs involved in interorgan regulation?

\section{HYPOTHESIS}

Recent studies have found that bone could affect multiple organs and be involved in physiological and pathological changes by secreting biomolecules $(14,15)$. Notably, EVs, 30-1,000 nm in diameter, which include exosomes and microvesicles, have been demonstrated to transfer functional proteins, mRNAs, and miRNAs to neighboring cells and serve as mediators of intercellular and long-distance communication (9, 16-18). Researchers have found that EVs could participate in interorgan communication and play an important role in physiological regulation. In addition, it was found that bone-related cells can also exchange genetic information and regulate bone remodeling through EVs $(19,20)$. Given these findings, we hypothesized that bone-related EVs may represent an important paradigm for remote organ effects.

\section{EVALUATION OF THE HYPOTHESIS}

\section{EVs Participate in Interorgan Communication}

With the deepening of research, investigators have paid attention to EVs as biomarkers in the early stage and have gradually discovered that EVs can stably transfer proteins and genetic material, which suggests that EVs may play a potential role in physiological regulation.

More importantly, the discovery of circulating EVs has given researchers new inspiration. Fruhbeis et al. found that exercise triggered a rapid release of EVs into the circulation. The dynamics of EVs varied between cycling and running exercise. The results suggest that EVs released during physical activity may participate in long-distance signaling during exercise-mediated adaptation processes (21). Guescini et al. found that muscle tissue could release EVs carrying muscle-specific miR-133b and miR-181a-5p under regular physical exercise (22). Thomou et al. determined that adipose tissue constitutes an important source of circulating exosomal miRNAs, which can regulate the expression of fibroblast growth factor 21 (FGF21) in liver tissues. These results indicate that adipose-derived exosomal miRNAs can regulate distant organs (23). Ying et al. also found that macrophages in adipose tissue secreted EVs, which could transfer miRNAs to insulin target cells. These EVs can modulate systemic insulin and glucose tolerance by directly affecting cellular insulin signaling (24). In addition to genetic materials, circulating exosomal proteins also have potential functions. Whitham et al. found that EVs liberated by exercise have a propensity to localize in the liver and can transfer their protein cargo (25). These results provide evidence for a new paradigm, namely, EV trafficking, which can act as a bridge in interorgan communication and exert systemic biological effects. More interestingly, Yoshida et al. found that EVs containing eNAMPT can delay aging and prolong life in mice. They also confirmed that eNAMPT in human plasma is mainly contained in EVs (26).

In addition to participating in energy metabolism, circulating exosomes have also been found to be widely involved in the physiological and pathological processes of the heart. Cheng et al. found that circulating myo-miRs are carried in exosomes and mediate functional crosstalk between the ischemic heart and the bone marrow (BM). They found a higher level of circulating exosomes in acute myocardial infarction (AMI) mice that could transfer myo-miRs into BM monocular cells (MNCs). The results also showed that AMI exosomes could mediate the downregulation of CXC chemokine receptor 4 (CXCR4) and contribute to BM progenitor cell mobilization (27). However, the function of circulating exosomes has not been entirely elucidated. These studies suggest that EVs transferring genetic cargos or proteins could constitute a previously undescribed class of regulators that control metabolism in distant tissues, providing a new mechanism of cell-cell crosstalk. Based on the above evidence, it is suggested that EV trafficking may be an important biological process of interorgan communication.

\section{BONE-SECRETED FACTORS PARTICIPATE IN INTERORGAN COMMUNICATION}

Recent studies have found that several bone-secreted factors participate in interorgan communication (Figure 1). Osteocalcin is an osteoblast-specific protein that is secreted at high levels in the bone extracellular matrix, and the genes encoding osteocalcin start to be expressed during development around the time bone mineralization begins (15). Many studies have shown that OCN plays a key role in the regulation of energy metabolism. For adipose tissue, OCN treatment could upregulate the expression of adiponectin, improve glucose uptake and insulin sensitivity in vivo and suppress the secretion of proinflammatory cytokines in adipocytes in vitro (28). OCN supports muscle function during exercise in part through the release of IL-6, the first myokine found to be rapidly released into the blood during exercise, enhancing glucose and fatty acid uptake into myofibers $(29,30)$. These observations and studies suggest that the effects of OCN on obesity and insulin resistance could be a result of its capacity to promote insulin sensitivity in the liver and adipose tissue, energy expenditure in muscle and insulin production in the pancreas and to upregulate expression of functional genes in the pancreas, muscle and adipose tissue. Male osteocalcin ${ }^{-/-}$ mice had low circulating levels of testosterone and bred poorly, which indicated that $\mathrm{OCN}$ may also regulate reproductive functions (5). Moreover, many unexpected observations showed that osteocalcin may influence the development of the brain and cognitive functions of the offspring. Osteocalcin is necessary 


\section{Bone-secreted factors regulate interorgan crosstalk}

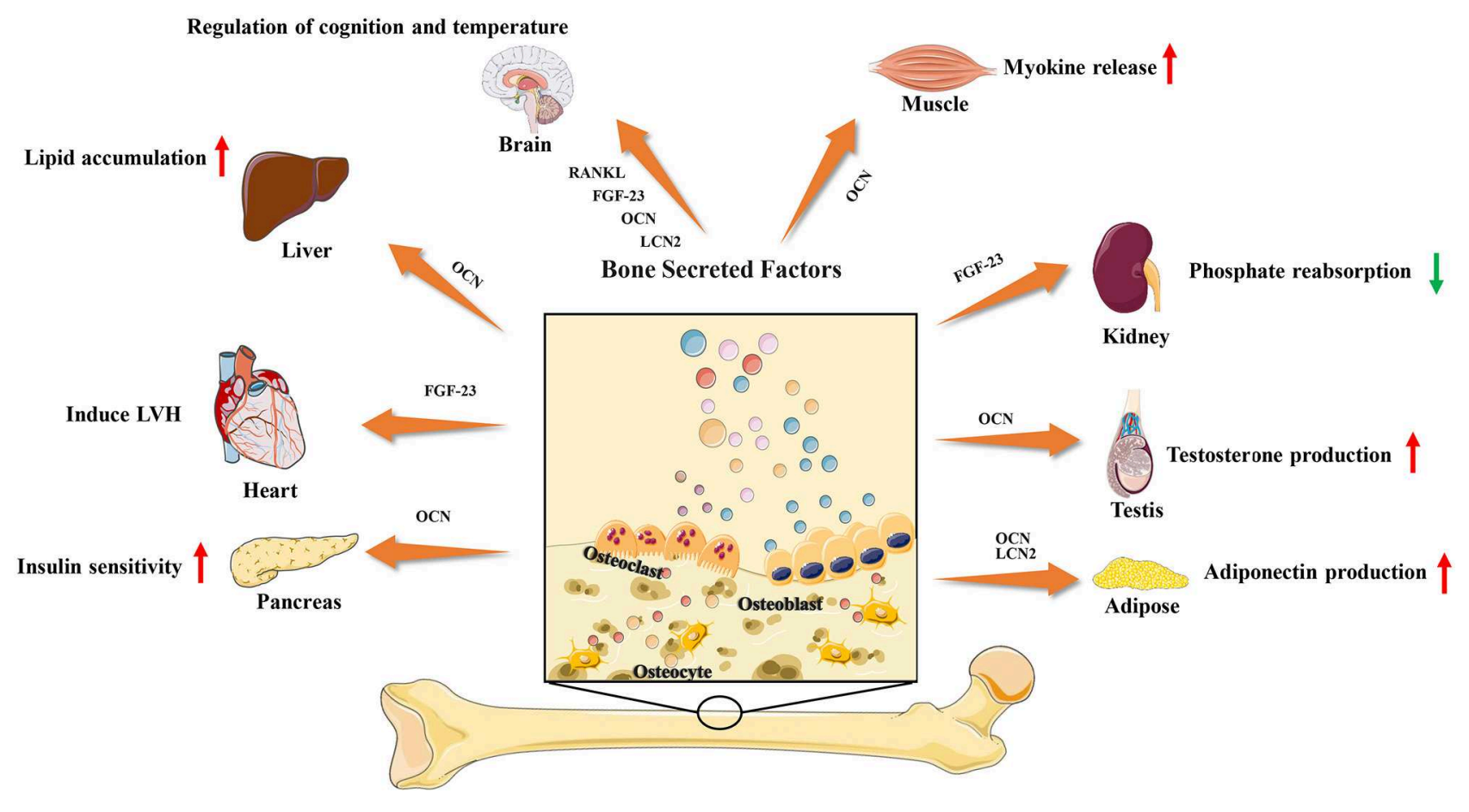

FIGURE 1 | Bone-secreted factors participate in interorgan communication.

and sufficient to correct age-related declines in cognitive function in mice. Intriguingly, the maternal production of osteocalcin appears to be necessary for normal fetal brain development $(5,31,32)$.

FGF23 is a phosphaturic hormone produced by osteocytes and osteoblasts. FGF23 mainly targets the renal proximal tubules to inhibit calcitriol production and the expression of the sodium/phosphate cotransporters NaPi2a and NaPi2c, thus inhibiting renal phosphate reabsorption $(1,2,33)$. Furthermore, recent studies have found that the increased level of FGF23 may induce left ventricular hypertrophy $(\mathrm{LVH})$ and is associated with a risk of mortality (34). However, several large epidemiological studies demonstrated a powerful dose-dependent association between serum levels of FGF23 and a higher risk of mortality in end-stage renal disease (ESRD) patients (35-37). These observations showed that FGF23 may connect bone with heart diseases and renal disorders. In addition to these two factors, bone-derived lipocalin2 (LCN2) was also found to suppress appetite and regulate fat mass, which indicated a potential connection between bone and brain $(38,39)$.

In recent years, researchers have found that RANK neurons are involved in the regulation of body temperature in the preoptic area of the hypothalamus. In stroke, the RANKLRANK signaling pathway protects neurons and reduces nerve damage. In autoimmune diseases, RANKL can participate in and assist Th17 cells in entering the brain parenchyma through the blood-brain barrier. In metabolic regulation, RANK signaling is involved in modulating Neuropeptide Y(NPY) levels and through that matching bone mass to body weight. The selective deletion of RANK from NPY neurons leads to a significant increase in fat mass and a decrease in whole body bone mineral density $(6,7,40-42)$.

As more and more bone-secreted factors were confirmed to be involved in interorgan communication, we can conclude that bone may actively participate in the regulation of physiological processes.

\section{BONE-SECRETED FACTORS TRANSFERRED BY EVs}

However, there is no definitive evidence to date that bone-derived EVs could regulate remote organs. Various studies have found that bone secreted factors involved in interorgan communication could be transferred through EVs. Yi et al. found that OCN could be packed into exosomes and is able to be transferred to the aorta endothelial cells via exosome incorporation (43). Previous studies have indicated that the serum level of OCN is inversely associated with atherosclerosis. However, the expression of $\mathrm{OCN}$ in endothelial progenitor cells (EPCs) has not been clarified. Importantly, this study indicated that OCN-exosomes could promote the proliferation of endothelial cells efficiently via OCNGPRC6A signaling. In addition, LCN2 was also found to be packed in EVs. Rollet-Cohen et al. compared the proteomic 
content of respiratory exosomes from cystic fibrosis, primary ciliary dyskinesia and asthma patients. The results showed high expression of LCN2 in exosomes from cystic fibrosis patients (44). The above studies suggest that EVs may contain bonederived factors and play a regulatory role in physiological or pathological conditions. Because current research has not elucidated the complete function of bone-derived EVs, further research should focus on the relationship between EVs and bone-derived factors.

\section{PARACRINE ROLE OF BONE-DERIVED EVs}

Bone is a dynamic organ that is constantly remodeled by the activities of several cells, including mesenchymal stem cells, osteoblasts, osteocytes, and osteoclasts. Many studies have found that bone-derived EVs play important roles in cellular communication in bone remodeling. In the bone-remodeling microenvironment, bone-derived EVs contain specific proteins and genetic material and are involved in regulating cell activity.

Bidirectional osteoblast-osteoclast communication plays crucial roles in bone remodeling (45). Cappariello et al. found that osteoblast-derived EVs are involved in intercellular communication and facilitate the function of osteoclasts. In osteoblasts pretreated with parathyroid hormone (PTH), EVs from osteoblasts contain RANKL in their outer membrane and are captured by osteoclasts. More importantly, PTH also increased the total number of RANKL-positive EVs. In addition, this study also showed that EVs are biotechnological tools to shuttle antiosteoclastic drugs such as dasatinib and zoledronate. To investigate the role of osteoblast-derived EVs, they used the $\mathrm{RANKL}^{-/-}$mice, a murine model characterized by the lack of osteoclasts. Osteoclasts in $\mathrm{RANKL}^{-/-}$mice were inactive due to the lack of pro-osteoclastic function. The osteoblast-derived EVs stimulated osteoclasts in $\mathrm{RANKL}^{/-}$mice. Osteoblast-derived EVs could recover this function by inducing TRAcp-positive cells $(46,47)$. On the other hand, osteoclast-derived EVs can also affect osteoblast function. Li et al. and Sun et al. showed that exosomal miR-214-3p from osteoclasts, which participated in the crosstalk of bone cells, inhibited osteoblast activity and reduced bone formation $(11,12)$. Mesenchymal stem cells (MSCs) are multipotent cells that can differentiate into bone-related cells. Therefore, MSCs are often used as seed cells for bone repair (48-51). However, the paracrine factors of MSCs, rather than exogenous MSCs themselves, were recently shown to play a major role in promoting tissue repair $(52,53)$. Recent studies have revealed that EVs derived from MSCs could transfer genetic material for cell communication, thereby regulating the main processes of bone regeneration, including osteogenesis and angiogenesis (19). Qin et al. found that MSC-derived EVs were endocytosed by osteoblasts and regulated their activity in vitro (54). MSC-derived EVs could also be captured by human umbilical-vein endothelial cells (HUVECs) and transmit miR494 to stimulate angiogenesis in vitro and in vivo (55). Likewise, endothelial cell-derived EVs could be taken up by MSCs and transmit miR-31 to inhibit osteogenic differentiation (56).
Sato et al. compared osteocyte-secreted exosomes and circulating exosomes from osteocyte-less mice. The results indicated that ablation of osteocytes in mice alters the miRNA levels of plasma exosomes (57). This study linked osteocyte exosomes to circulating exosomes, which provides the concept that osteocyte-derived exosomes may transfer their components to other organs.

In conclusion, paracrine functions of bone-derived EVs are widely involved in intercellular communication and genetic material transmission in the bone microenvironment.

\section{MAJOR QUESTIONS REMAIN IN VERIFYING THIS HYPOTHESIS}

There are still many key issues that need to be addressed to prove this hypothesis. First, current research has confirmed that bone secreted factors play a role in the regulation of brain, liver, muscle, pancreas, testis, and adipose tissue. Further research should begin with these known directions to explore whether bone-related EVs are involved in the regulation of these organs. Second, it is also important to identify the EV-mediated bone effects on the physiological functions of other organs. Current research has primarily explored the role of bone in endocrine and energy metabolism, but whether bone has effects on other systems, such as the immune system, requires further exploration.

Another important problem is the lack of knowledge of bonederived EVs. Most studies published thus far have analyzed mixed EV populations, and the most important step is to comprehensively compare the different subtypes of EVs (58). Furthermore, specific markers or characteristics of EVs from different cells are also needed for identification. To determine the roles of bone-derived EVs in interorgan communication, researchers need to identify the subtypes and characteristics of EVs from bone-related cells and determine whether different subtypes have specific functions or even prominent target organs. Furthermore, important questions remain regarding the spatiotemporal properties of EVs in vivo (18). Currently, many studies have shown the effects of EVs on target cells, but most studies are performed in vitro or in vivo by injection of EVs harvested from cells in culture. The characteristics of bone-derived EVs in physiological states require further exploration. Further study should illuminate the life span, the concentration and the in vivo metabolism of bone-derived EVs in the bloodstream. As a result, these studies may not reflect the spatiotemporal properties or concentrations of EVs active in normal physiology in vivo. A direct demonstration that functional EV-mediated biological cargo transfer is the relevant mechanism in certain biological processes is still difficult to achieve (59).

\section{IMPORTANCE OF CONFIRMING THIS HYPOTHESIS}

Confirmation of EV-mediated interorgan communication of bone would help deepen the understanding of the communication networks between the skeleton and other 
systems. The results of existing research have shown that bonesecreted proteins could regulate interorgan communication. The growing awareness that bone is an active organ will broaden our understanding of the pathogenesis of bone in other systemic diseases. The interorgan communication function of bone mediated by EVs may become a therapeutic target for other diseases.

Furthermore, confirming this hypothesis may expand our knowledge of EVs. Many studies have confirmed the widespread presence of EVs in body fluids. However, the biological functions of biofluid-derived EVs remain unclear. The current study mainly focused on EVs from body fluids for disease diagnosis, but their physiological functions still need to be revealed. In addition, the role of EVs is not only transferring functional proteins but also transferring genetic material. Confirmation of the regulation of bone-secreted EVs on multiple organs will help us better understand the transmission of genetic information. Meanwhile, as EVs have been acknowledged as

\section{REFERENCES}

1. Hu MC, Shiizaki K, Kuro-o M, Moe OW. Fibroblast growth factor 23 and Klotho: physiology and pathophysiology of an endocrine network of mineral metabolism. Annu Rev Physiol. (2013) 75:503-33. doi: 10.1146/annurev-physiol-030212-183727

2. Quarles LD. Skeletal secretion of FGF-23 regulates phosphate and vitamin D metabolism Nat Rev Endocrinol. (2012) 8:276-86. doi: 10.1038/nrendo.2011.218

3. Mizokami A, Kawakubo-Yasukochi T, Hirata M. Osteocalcin and its endocrine functions. Biochem. Pharmacol. (2017) 132:1-8. doi: 10.1016/j.bcp.2017.02.001

4. Mosialou I, Shikhel S, Liu J, Maurizi A, Luo N, He Z, et al. MC4R-dependent suppression of appetite by bone-derived lipocalin 2. Nature. (2017) 543:38590. doi: 10.1038/nature21697

5. Oury F, Khrimian L, Denny CA, Gardin A, Chamouni A, Goeden N, et al. Maternal and offspring pools of osteocalcin influence brain development and functions. Cell. (2013) 155:228-41. doi: 10.1016/j.cell.2013.08.042

6. Lee NJ, Clarke IM, Zengin A, Enriquez RF, Nagy V, Penninger JM, et al. RANK deletion in neuropeptide $\mathrm{Y}$ neurones attenuates oestrogen deficiency-related bone loss. J. Neuroendocrinol. (2019) 31:e12687. doi: 10.1111/jne.12687

7. Shimamura M, Nakagami H, Osako MK, Kurinami H, Koriyama H, Zhengda $\mathrm{P}$, et al. OPG/RANKL/RANK axis is a critical inflammatory signaling system in ischemic brain in mice. Proc Natl Acad Sci USA. (2014) 111:8191-6. doi: 10.1073/pnas.1400544111

8. Martínez MC, Andriantsitohaina R. Extracellular vesicles in metabolic syndrome. Circ Res. (2017) 120:10-1674. doi: 10.1161/CIRCRESAHA.117.309419

9. Boyiadzis $M$, Whiteside TL. The emerging roles of tumor-derived exosomes in hematological malignancies. Leukemia. (2017) 31:1259-68. doi: 10.1038/leu.2017.91

10. Zappulli V, Friis KP, Fitzpatrick Z, Maguire CA, Breakefield XO. Extracellular vesicles and intercellular communication within the nervous system. J. Clin. Invest. (2016) 126:4-1198. doi: 10.1172/JCI81134

11. Li D, Liu J, Guo B, Liang C, Dang L, Lu C, et al. Osteoclast-derived exosomal miR-214-3p inhibits osteoblastic bone formation. Nat. Commun. (2016) 7:10872. doi: 10.1038/ncomms10872

12. Sun W, Zhao C, Li Y, Wang L, Nie G, Peng J, et al. Osteoclastderived microRNA-containing exosomes selectively inhibit osteoblast activity. Cell Disc. (2016) 2:16015. doi: 10.1038/celldisc. 2016.15

13. Morhayim J, van de Peppel J, Braakman E, Rombouts EWJC, ter Borg MND, Dudakovic A, et al. Osteoblasts secrete miRNA-containing extracellular natural targeted-drug carriers, EV-based nanotechnology has provided unprecedented opportunities to help develop EVrelated therapeutics $(60,61)$. Research into the communication between skeleton-related EVs and other organs may identify novel therapeutic targets for medical intervention.

\section{AUTHOR CONTRIBUTIONS}

LZ and PT made major contributions to the conception of the work. YL and PY drafted the manuscript. ZG, MC, HL,YD, and YG revised the manuscript.

\section{FUNDING}

This article was funded by the National Natural Science Foundation of China (81702176 and 81772369). The original elements used in the figures are from Servier Medical Art (http:// smart.servier.com/). vesicles that enhance expansion of human umbilical cord blood cells. Sci Rep (2016) 6:32034. doi: 10.1038/srep32034

14. Han Y, You X, Xing W, Zhang Z, Zou W. Paracrine and endocrine actions of bone-the functions of secretory proteins from osteoblasts, osteocytes, and osteoclasts. Bone Res. (2018) 6:16. doi: 10.1038/s41413-018-0019-6

15. Karsenty G, Olson EN. Bone and muscle endocrine functions: unexpected paradigms of inter-organ communication. Cell. (2016) 164:6-1248. doi: $10.1016 /$ j.cell.2016.02.043

16. Tsatsaronis JA, Franch-Arroyo S, Resch U, Charpentier E. Extracellular vesicle RNA: a universal mediator of microbial communication? Trends Microbiol. (2018) 26:5-401. doi: 10.1016/j.tim.2018.02.009

17. Becker A, Thakur BK, Weiss JM, Kim HS, Peinado H, Lyden D. Extracellular vesicles in cancer: cell-to-cell mediators of metastasis. Cancer Cell. (2016) 30:6-836. doi: 10.1016/j.ccell.2016.10.009

18. Maas SLN, Breakefield XO, Weaver AM. Extracellular vesicles: unique intercellular delivery vehicles. Trends Cell Biol. (2017) 27:3-172. doi: $10.1016 /$ j.tcb.2016.11.003

19. Yin P, Li Y, Lv H, Deng Y, Meng Y, Zhang L, et al. Exchange of genetic material: a new paradigm in bone cell communications. Cell Mol Life Sci. (2018) 75:11-1989. doi: 10.1007/s00018-018-2782-3

20. Xie Y, Chen Y, Zhang L, Ge W, Tang P. The roles of bone-derived exosomes and exosomal microRNAs in regulating bone remodelling. J Cell Mol Med. (2017) 21:5-1033. doi: 10.1111/jcmm.13039

21. Fruhbeis C, Helmig S, Tug S, Simon P, Kramer-Albers EM. Physical exercise induces rapid release of small extracellular vesicles into the circulation. $J$ Extracell Vesicles. (2015) 4:28239. doi: 10.3402/jev.v4.28239

22. Guescini M, Canonico B, Lucertini F, Maggio S, Annibalini G, Barbieri E, et al. Muscle Releases alpha-sarcoglycan positive extracellular vesicles carrying miRNAs in the bloodstream. PLoS ONE. (2015) 10:e0125094. doi: 10.1371/journal.pone.0125094

23. Thomou T, Mori MA, Dreyfuss JM, Konishi M, Sakaguchi M, Wolfrum C, et al. Adipose-derived circulating miRNAs regulate gene expression in other tissues. Nature. (2017) 542:7642-450. doi: 10.1038/nature21365

24. Ying W, Riopel M, Bandyopadhyay G, Dong Y, Birmingham A, Seo JB, et al. Adipose tissue macrophage-derived exosomal mirnas can modulate in vivo and in vitro insulin sensitivity. Cell. (2017) 171:2-372.e12. doi: 10.1016/j.cell.2017.08.035

25. Whitham M, Parker BL, Friedrichsen M, Hingst JR, Hjorth M, Hughes WE, et al. Extracellular vesicles provide a means for tissue crosstalk during exercise. Cell Metab. (2018) 27:1-237.e4. doi: 10.1016/j.cmet.2017.12.001

26. Yoshida M, Satoh A, Lin JB, Mills KF, Sasaki Y, Rensing N, et al. Extracellular vesicle-contained eNAMPT delays aging and extends lifespan in mice. Cell Metab. (2019) 30:2-329.e5. doi: 10.1016/j.cmet.2019.05.015 
27. Cheng M, Yang J, Zhao X, Zhang E, Zeng Q, Yu Y, et al. Circulating myocardial microRNAs from infarcted hearts are carried in exosomes and mobilise bone marrow progenitor cells. Nat Commun. (2019) 10:959. doi: 10.1038/s41467-019-08895-7

28. Guedes J, Esteves JV, Morais MR, Zorn TM, Furuya DT. Osteocalcin improves insulin resistance and inflammation in obese mice: participation of white adipose tissue and bone. Bone. (2018) 115:68-82. doi: 10.1016/j.bone.2017.11.020

29. Mera P, Laue K, Ferron M, Confavreux C, Wei J, Galán-Díez M, et al. Osteocalcin signaling in myofibers is necessary and sufficient for optimum adaptation to exercise. Cell Metab. (2016) 23:6-1078. doi: 10.1016/j.cmet.2016.05.004

30. Yoshikawa Y, Kode A, Xu L, Mosialou I, Silva BC, Ferron M, et al. Genetic evidence points to an osteocalcin-independent influence of osteoblasts on energy metabolism. J Bone Miner Res. (2011) 26:9-2012. doi: $10.1002 / \mathrm{jbmr} .417$

31. Obri A, Khrimian L, Karsenty G, Oury F. Osteocalcin in the brain: from embryonic development to age-related decline in cognition. Nat Rev Endocrinol. (2018) 14:3-174. doi: 10.1038/nrendo.2017.181

32. Ferron M, Lacombe J. Regulation of energy metabolism by the skeleton: osteocalcin and beyond. Arch Biochem Biophys. (2014) 561:137-46. doi: 10.1016/j.abb.2014.05.022

33. Erben RG. Pleiotropic actions of FGF23. Toxicol Pathol. (2017) 45:904-10. doi: $10.1177 / 0192623317737469$

34. Andrukhova O, Slavic S, Odörfer KI, Erben RG. Experimental myocardial infarction upregulates circulating fibroblast growth factor-23. J Bone Miner Res. (2015) 30:10-1831. doi: 10.1002/jbmr.2527

35. Wolf M. The biomarker niche for fibroblast growth factor 23 testing in CKD. J Am Soc Nephrol. (2015) 26:1-7. doi: 10.1681/ASN.2014060621

36. Scialla JJ, Xie H, Rahman M, Anderson AH, Isakova T, Ojo A, et al. Fibroblast growth factor-23 and cardiovascular events in CKD. J Am Soc Nephrol. (2014) 25:2-349. doi: 10.1681/ASN.2013050465

37. Jüppner $H$, Wolf $M$, Salusky IB. FGF-23: more than a regulator of renal phosphate handling? J Bone Miner Res. (2010) 25:10-2091. doi: $10.1002 / \mathrm{jbmr} .170$

38. Palmiter RD. Bone-derived hormone suppresses appetite. Nature. (2017) 543:7645-320. doi: 10.1038/nature21501

39. Jansson J, Palsdottir V, Hägg DA, Schéle E, Dickson SL, Anesten F, et al. Body weight homeostat that regulates fat mass independently of leptin in rats and mice. Proc Natl Acad Sci USA. (2018) 115:2-427. doi: $10.1073 /$ pnas.1715687114

40. Lee NJ, Clarke IM, Enriquez RF, Nagy V, Penninger J, Baldock PA, et al. Central RANK signalling in NPY neurons alters bone mass in male mice. Neuropeptides. (2018) 68:75-83. doi: 10.1016/j.npep.2018.02.004

41. Hanada R, Leibbrandt A, Hanada T, Kitaoka S, Furuyashiki T, Fujihara H, et al. Central control of fever and female body temperature by RANKL/RANK. Nature. (2009) 462:7272-505. doi: 10.1038/nature08596

42. Guerrini MM, Okamoto K, Komatsu N, Sawa S, Danks L, Penninger JM, et al. Inhibition of the TNF family cytokine RANKL prevents autoimmune inflammation in the central nervous system. Immunity. (2015) 43:6-1174. doi: 10.1016/j.immuni.2015.10.017

43. Yi M, Wu Y, Long J, Liu F, Liu Z, Zhang YH, et al. Exosomes secreted from osteocalcin-overexpressed endothelial progenitor cells promoted endothelial cell angiogenesis. Am J Physiol Cell Physiol. (2019) 317:C932-41. doi: 10.1152/ajpcell.00534.2018

44. Rollet-Cohen V, Bourderioux M, Lipecka J, Chhuon C, Jung VA, Mesbahi $\mathrm{M}$, et al. Comparative proteomics of respiratory exosomes in cystic fibrosis, primary ciliary dyskinesia and asthma. Proteomics J. (2018) 185:1-7. doi: 10.1016/j.jprot.2018.07.001

45. Liu M, Sun Y, Zhang Q. Emerging role of extracellular vesicles in bone remodeling. J Dent Res. (2018) 97:8-859. doi: 10.1177/0022034518764411
46. Collison J. Bone: extracellular vesicles in bone cell crosstalk. Nat Rev Rheumatol. (2018) 14:1-2. doi: 10.1038/nrrheum.2017.191

47. Cappariello A, Loftus A, Muraca M, Maurizi A, Rucci N, Teti A. Osteoblastderived extracellular vesicles are biological tools for the delivery of active molecules to bone. J Bone Miner Res. (2018) 33:3-517. doi: 10.1002/jbmr.3332

48. Crane JL, Cao X. Bone marrow mesenchymal stem cells and TGF- $\beta$ signaling in bone remodeling. J Clin Invest. (2014) 124:2-466. doi: 10.1172/JCI70050

49. Zhang ZY, Teoh SH, Hui JH, Fisk NM, Choolani M, Chan JK. The potential of human fetal mesenchymal stem cells for off-theshelf bone tissue engineering application. Biomaterials. (2012) 33:9-2656. doi: 10.1016/j.biomaterials.2011.12.025

50. Undale AH, Westendorf JJ, Yaszemski MJ, Khosla S. Mesenchymal stem cells for bone repair and metabolic bone diseases. Mayo Clin Proc. (2009) 84:10-893. doi: 10.1016/S0025-6196(11)60506-5

51. Muruganandan S, Roman AA, Sinal CJ. Adipocyte differentiation of bone marrow-derived mesenchymal stem cells: cross talk with the osteoblastogenic program. Cell Mol Life Sci. (2009) 66:2-236. doi: 10.1007/s00018-008-8429-z

52. Veronesi F, Borsari V, Sartori M, Orciani M, Mattioli-Belmonte M, Fini M. The use of cell conditioned medium for musculoskeletal tissue regeneration. $J$ Cell Physiol. (2018) 233:6-4423. doi: 10.1002/jcp.26291

53. Phinney DG, Pittenger MF. Concise review: MSC-derived exosomes for cell-free therapy. Stem Cells. (2017) 35:4-851. doi: 10.1002/stem.2575

54. Qin Y, Wang L, Gao Z, Chen G, Zhang C. Bone marrow stromal/stem cellderived extracellular vesicles regulate osteoblast activity and differentiation in vitro and promote bone regeneration in vivo. Sci Rep. (2016) 6:21961. doi: 10.1038/srep21961

55. Nakamura $\mathrm{Y}$, Miyaki S, Ishitobi $\mathrm{H}$, Matsuyama S, Nakasa T, Kamei N, et al. Mesenchymal-stem-cell-derived exosomes accelerate skeletal muscle regeneration. FEBS Lett. (2015) 589:11-1257. doi: 10.1016/j.febslet.2015.03.031

56. Weilner S, Schraml E, Wieser M, Messner P, Schneider K, Wassermann K, et al. Secreted microvesicular miR-31 inhibits osteogenic differentiation of mesenchymal stem cells. Aging Cell. (2016) 15:4-744. doi: 10.1111/acel.12484

57. Sato M, Suzuki T, Kawano M, Tamura M. Circulating osteocyte-derived exosomes contain miRNAs which are enriched in exosomes from MLO-Y4 cells. Biomed. Rep. (2017) 6:2-223. doi: 10.3892/br.2016.824

58. Tkach M, Thery C. Communication by extracellular vesicles: where we are and where we need to go. Cell. (2016) 164:6-1226. doi: 10.1016/j.cell.2016. 01.043

59. Yin P, Lv H, Li Y, Deng Y, Zhang L, Tang P. Exosome-mediated genetic information transfer, a missing piece of osteoblast-osteoclast communication puzzle. Front Endocrinol. (2017) 8:336. doi: 10.3389/fendo.2017. 00336

60. Conlan RS, Pisano S, Oliveira MI, Ferrari M, Mendes Pinto I. Exosomes as reconfigurable therapeutic systems. Trends Mol Med. (2017) 23:7-636. doi: 10.1016/j.molmed.2017.05.003

61. Malda J, Boere J, van de Lest CH, van Weeren P, Wauben MH. Extracellular vesicles - new tool for joint repair and regeneration. Nat Rev Rheumatol. (2016) 12:4-243. doi: 10.1038/nrrheum.2015.170

Conflict of Interest: The authors declare that the research was conducted in the absence of any commercial or financial relationships that could be construed as a potential conflict of interest.

Copyright (c) $2019 \mathrm{Li}$, Yin, Guo, Lv, Deng, Chen, Gu, Tang and Zhang. This is an open-access article distributed under the terms of the Creative Commons Attribution License (CC BY). The use, distribution or reproduction in other forums is permitted, provided the original author(s) and the copyright owner(s) are credited and that the original publication in this journal is cited, in accordance with accepted academic practice. No use, distribution or reproduction is permitted which does not comply with these terms. 